\title{
ORBITAL-DEPENDENT DENSITY FUNCTIONALS FOR CHEMICAL CATALYSIS
}

DONALD TRUHLAR

REGENTS OF THE UNIVERSITY OF MINNESOTA MINNEAPOLIS

$10 / 17 / 2014$

Final Report 


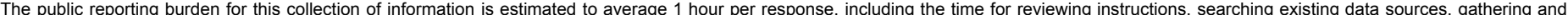

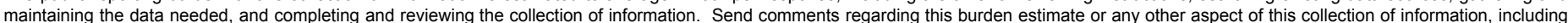

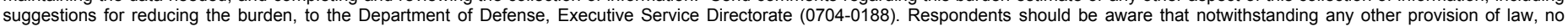
person shall be subject to any penalty for failing to comply with a collection of information if it does not display a currently valid OMB control number

PLEASE DO NOT RETURN YOUR FORM TO THE ABOVE ORGANIZATION.
1. REPORT DATE (DD-MM-YYYY)
2. REPORT TYPE
14-09-2014
Final Report
3. DATES COVERED (From - To)
6-15-2011 to 6-14-2014

\section{TITLE AND SUBTITLE}

ORBITAL-DEPENDENT DENSITY FUNCTIONALS FOR CHEMICAL CATALYSIS 5a. CONTRACT NUMBER

FA9550-11-1-0078

\section{5b. GRANT NUMBER}

FA9550-11-1-0078

5c. PROGRAM ELEMENT NUMBER

does not apply

\section{AUTHOR(S)}

Truhlar, Donald G

5d. PROJECT NUMBER

does not apply 5e. TASK NUMBER

does not apply

5f. WORK UNIT NUMBER

does not apply

8. PERFORMING ORGANIZATION REPORT NUMBER

does not apply

University of Minnesota

207 Pleasant Street SE

Minneapolis, MN 55455-0431

9. SPONSORING/MONITORING AGENCY NAME(S) AND ADDRESS(ES)

Air Force Office of Scientific Research

10. SPONSOR/MONITOR'S ACRONYM(S)

AFOSR

11. SPONSOR/MONITOR'S REPORT NUMBER(S)

does not apply

\section{DISTRIBUTION/AVAILABILITY STATEMENT}

UU

\section{SUPPLEMENTARY NOTES}

none

\section{ABSTRACT}

Catalyzed reactions are important for synthesizing new materials, for energy production and conversion, and for electrochemical, photochemical, and solar cells and devices. The objective of the preroject was to develop better orbital-dependent density functionals and improved algorithms for applications of computational chemistry to catalysis. The technical approach wass to propose new functional forms for the density functional and new algorithms for applying them and to test and validate those functionals and algorithms by applications to databases of experimental and accurate theoretical data and to important practical problems in current catalytic research. At a fundamental level we continued to explore the questions of what functional dependences must be present in the exchange-correlation energy for density functional theory to have improved predictive ability for catalysis involving transition metals. The rest was that we developed improved methods for modeling catalysis, for improved catalytic ligand design, and for understanding and predicting selectivity and specificity in catalytic reactions.

\section{SUBJECT TERMS}

theoretical and computational chemistry; physical chemistry; chemical physics; density functional theory; catalysis

\begin{tabular}{|c|c|c|}
\hline \multicolumn{3}{|c|}{ 16. SECURITY CLASSIFICATION OF: } \\
\hline $\begin{array}{c}\text { a. REPORT } \\
\text { UU }\end{array}$ & b. ABSTRACT & c. THIS PAGE \\
& UU & UU \\
\hline
\end{tabular}

$\begin{gathered}\text { 17. LIMITATION OF } \\ \text { ABSTRACT } \\ \text { UU }\end{gathered}$

8. NUMBER
OF
PAGES

19a. NAME OF RESPONSIBLE PERSON

Donald g. Truhlar

19b. TELEPHONE NUMBER (Include area code) 612-524-7555 
1. REPORT DATE. Full publication date, including day, month, if available. Must cite at least the year and be Year 2000 compliant, e.g. 30-06-1998; xx-06-1998; xx-xx-1998.

2. REPORT TYPE. State the type of report, such as final, technical, interim, memorandum, master's thesis, progress, quarterly, research, special, group study, etc.

3. DATES COVERED. Indicate the time during which the work was performed and the report was written, e.g., Jun 1997 - Jun 1998; 1-10 Jun 1996; May - Nov 1998; Nov 1998.

4. TITLE. Enter title and subtitle with volume number and part number, if applicable. On classified documents, enter the title classification in parentheses.

5a. CONTRACT NUMBER. Enter all contract numbers as they appear in the report, e.g. F33615-86-C-5169.

5b. GRANT NUMBER. Enter all grant numbers as they appear in the report, e.g. AFOSR-82-1234.

5c. PROGRAM ELEMENT NUMBER. Enter all program element numbers as they appear in the report, e.g. $61101 \mathrm{~A}$.

5d. PROJECT NUMBER. Enter all project numbers as they appear in the report, e.g. 1F665702D1257; ILIR.

5e. TASK NUMBER. Enter all task numbers as they appear in the report, e.g. 05; RF0330201; T4112.

5f. WORK UNIT NUMBER. Enter all work unit numbers as they appear in the report, e.g. 001; AFAPL30480105.

6. AUTHOR(S). Enter name(s) of person(s) responsible for writing the report, performing the research, or credited with the content of the report. The form of entry is the last name, first name, middle initial, and additional qualifiers separated by commas, e.g. Smith, Richard, J, Jr.

\section{PERFORMING ORGANIZATION NAME(S) AND} ADDRESS(ES). Self-explanatory.
8. PERFORMING ORGANIZATION REPORT NUMBER. Enter all unique alphanumeric report numbers assigned by the performing organization, e.g. BRL-1234; AFWL-TR-85-4017-Vol-21-PT-2.

\section{SPONSORING/MONITORING AGENCY NAME(S)} AND ADDRESS(ES). Enter the name and address of the organization(s) financially responsible for and monitoring the work.

10. SPONSOR/MONITOR'S ACRONYM(S). Enter, if available, e.g. BRL, ARDEC, NADC.

\section{SPONSOR/MONITOR'S REPORT NUMBER(S).} Enter report number as assigned by the sponsoring/ monitoring agency, if available, e.g. BRL-TR-829; -215.

12. DISTRIBUTION/AVAILABILITY STATEMENT. Use agency-mandated availability statements to indicate the public availability or distribution limitations of the report. If additional limitations/ restrictions or special markings are indicated, follow agency authorization procedures, e.g. RD/FRD, PROPIN, ITAR, etc. Include copyright information.

13. SUPPLEMENTARY NOTES. Enter information not included elsewhere such as: prepared in cooperation with; translation of; report supersedes; old edition number, etc.

14. ABSTRACT. A brief (approximately 200 words) factual summary of the most significant information.

15. SUBJECT TERMS. Key words or phrases identifying major concepts in the report.

16. SECURITY CLASSIFICATION. Enter security classification in accordance with security classification regulations, e.g. U, C, S, etc. If this form contains classified information, stamp classification level on the top and bottom of this page.

17. LIMITATION OF ABSTRACT. This block must be completed to assign a distribution limitation to the abstract. Enter UU (Unclassified Unlimited) or SAR (Same as Report). An entry in this block is necessary if the abstract is to be limited. 


\section{Final report}

Air Force Office of Scientific Research (AFOSR) grant no. FA9550-11-1-0078.

Title: Orbital-Dependent Density Functionals for Chemical Catalysis

Dates of project: 6/15/11-6/14/14 (3 years)

\section{Report}

We achieved considerable success in developing new kinds of density functionals and new density functionals for catalysis. We also made several successful applications of density functional theory.

As a long-standing puzzle, experimental observations reveal faster organophosphine dissociation in the olefin metathesis by Grubbs' s firstgeneration precatalyst (Gen I) than by the second-generation precatalyst (Gen II), but Gen I shows less catalytic activity. We showed by electronic structure calculations with the M06-L density functional that carbene rotamer energetic effects are responsible for the inverse relation between organophosphine dissociation rate and catalytic activity. The carbene rotamer acts as a toggle switch, triggering the dissociative mechanism that produces the active catalyst. The slower catalyst production in Gen II as compared to Gen I is not a pure electronic effect but results from rotameric coupling to the dissociation coordinate speeding up Gen I dissociation more than Gen II dissociation. If organophosphine dissociation were to occur with fixed rotamer orientation, Gen II would be produced faster than Gen I, as originally expected. The rotameric energetics also contributes to the higher catalytic activity of the Gen II catalyst. For molecules containing the fourth-period element arsenic, we test (i, ii) the accuracy of all-electron (AE) basis sets from the def2-x ZVP and ma-x ZVP series (where $x Z$ is $S, T Z$, or $Q Z$ ), (iii) the accuracy of the 6-311G series of $A E$ basis sets with additional polarization and diff use functions, and (iv) the performance of effective core potentials (ECPs). The first set of tests involves basis-set convergence studies with eleven density functionals for five cases: equilibrium dissociation energy (De ) of As2, vertical ionization potential (VIP) of As2, IP of As, acid dissociation of $\mathrm{H} 3 \mathrm{AsO} 4$, and De of FeAs. A second set of tests involves the same kinds of basis-set convergence studies for the VIP and De values of As3 and As4 clusters. Both relativistic and nonrelativistic calculations are considered, including in each case both $A E$ calculations and calculations with ECPs. Convergence and accuracy are assessed by comparing to relativistic AE calculations with the cc-pV5Z-DK or ma-cc-pV5Z-DK basis and to nonrelativistic AE calculations with the cc-pV5Z or ma-cc-pV5Z basis. The primary objective of this study is to evaluate the abilities of ECPs with both their recommended basis sets and other basis sets to reproduce the results of allelectron relativistic calculations. The performance of the def2 and ma series basis sets is consistent with their sizes, and quadruple- $\zeta$ basis sets are the best. 
The def2-TZVP basis set performs better than most of the 6-311G series basis sets, which are the most commonly used basis sets in the previous studies of arsenic compounds. However, relativistic def2-TZVP calculations are not recommended. The large-core ECPs, which are the only available ECPs for arsenic in the popular Gaussian program, have average errors of $9 \_12 \mathrm{kcal} / \mathrm{mol}$ for the arsenic systems studied; therefore, these ECPs are not recommended. The triple- $\zeta$ small-core relativistic ECP (RECP) basis set cc-pVTZ-PP is found to have performance better than that of the def2-TZVP basis set, and it is highly recommended for arsenic-containing systems. The double- $\zeta$ RECP basis set masc-SVP is recommended for large arsenic systems. The double- $\zeta$ RECP basis set ma-sc-SVP is recommended for large arsenic systems for which the def2TZVP and cc-pVTZ-PP basis sets are unaffordable, if a basis-set error of $\sim 2$ $\mathrm{kcal} / \mathrm{mol}$ can be tolerated.

The Gibbs energy of reaction of oxidative addition of ammonia to an iridium complex in diethyl ether was calculated by seven density functional methods, in particular B3LYP, PBE, CAMB3LYP, M05, M06, M06-L, and oB97X. The calculated free energies, based on geometry optimization and frequency calculations in both the gas phase and solution, were compared with the experimental result, $-1.3 \mathrm{kcal} / \mathrm{mol}$, obtained by Hartwig and coworkers. The M06$L$ method gives the best result: $-1.4 \mathrm{kcal} / \mathrm{mol}$.

We employed noncollinear density functional theory to show that the low-spin state of Mn3 in a model of the oxygen-evolving complex of photosystem II avoids frustrated spin coupling by adopting a noncollinear arrangement of spins, thereby lowering the energy by $7 \mathrm{kcal} / \mathrm{mol}$. The high-spin state also has noncollinear spins. The optimum self-consistent field solutions for this multinuclear oxomanganese complex correspond to states that cannot be described by the unrestricted Slater determinants used in Kohn-Sham collinear density functional methods. This kind of spin coupling can be important in many open-shell systems, and the conventional collinear spin interpretation of chemical bonding in such systems should be viewed with caution.

The Minnesota family of exchange-correlation functionals, which consists of meta generalized gradient approximations (meta-GGAs) and global-hybrid metaGGAs, has been successful for density functional calculations of molecular structure, properties, and thermochemistry, kinetics, noncovalent interactions, and spectroscopy. Here, we generalize the functional form by using rangeseparated hybrid meta-GGA exchange. We optimized a functional, called M11, with the new form against a broad database of energetic chemical properties and compare its performance to that of several other functionals, including previous Minnesota functionals. We required the percentage of Hartree-Fock exchange to be 100 at large interelectronic distance, and we found an optimum percentage of 42.8 at short range. M11 has good across-the-board performance and the smallest mean unsigned error over the whole test set of 332 data; it has especially good performance for main-group atomization energies, proton 
affinities, electron affinities, alkyl bond dissociation energies, barrier heights, noncovalent interaction energies, and charge-transfer electronic excitation.

The performance of popular Hartree-Fock-based effective core potentials in Hartree-Fock and density functional calculations of $3 d$ transition metals was evaluated by basis-set convergence studies for ten cases: the equilibrium bond dissociation energy $\left(D_{e}\right)$ for dissociation of ground-state $\mathrm{Ti}_{2}$ to ground and excited atoms, the ground-state dissociation energies of $\mathrm{FeO}, \mathrm{Cu}_{2}, \mathrm{ScH}, \mathrm{TiH}, \mathrm{Sc}_{2}, \mathrm{Fe}_{2}$, and $\mathrm{TiV}^{+}$, and the first excitation energy of $\mathrm{Ti}$ atom. Each case was studied with 11 or 13 density functionals. For comparison, the accuracy of the all-electron def2-TZVP basis set is tested with both relativistic and nonrelativistic treatments. Convergence and accuracy are assessed by comparing to relativistic all-electron calculations with a nearly complete relativistic basis set (NCBS-DK, which denotes the cc-pV5Z-DK basis set for $3 \mathrm{~d}$ metals and hydrogen and the ma-ccpV5Z-DK basis set for oxygen) and to nonrelativistic all-electron calculations with a nearly complete nonrelativistic basis set (NCBS-NR, which denotes the ccpV5Z basis set for $3 d$ metals and hydrogen and the ma-cc-pV5Z basis set for oxygen). As compared to NCBS-DK results, all ECP calculations perform worse than def2-TZVP all-electron relativistic calculations when averaged over all 130 data (13 functionals and ten test cases). The compact effective potential (CEP) relativistic effective core potential (RECP) combined with a valence basis set developed for the many-electron Dirac_Fock (MDF10) RECP performs best in effective core potential calculations and has an average basis-set incompleteness error of $3.7 \mathrm{kcal} / \mathrm{mol}$, which is much larger than that $(0.9$ $\mathrm{kcal} / \mathrm{mol}$ ) of def2- TZVP relativistic all-electron results. Hence, the def2-TZVP relativistic all-electron calculations are recommended for accurate DFT calculations on $3 d$ transition metals. In addition to our general findings, we observed that all kinds of density functionals do not show the same trends. For example, when ECPs are used with hybrid functionals, which sometimes are not recommended for calculations of transition metal systems, they are found to perform better at achieving the basis-set limit than when used with local functionals and meta-GGA functionals. The most successful combination of RECP and basis set has a basis-set incompleteness error of $1.7-2.4 \mathrm{kcal} / \mathrm{mol}$ for hybrid generalized gradient approximations, which is smaller than that of nonrelativistic NCBS calculations (whose average basis-set incompleteness error for hybrid functionals is $2.7-2.9 \mathrm{kcal} / \mathrm{mol}$ ). The average basis-set incompleteness.

We reported a new local exchange-correlation energy functional that has significantly improved across-the-board performance, including main-group and transition metal chemistry and solid-state physics, especially atomization energies, ionization potentials, barrier heights, noncovalent interactions, isomerization energies of large molecules, and solid-state lattice constants and cohesive energies.

The ability of density functional theory to predict the relative energies of different spin states, especially for systems containing transition metal atoms, is of great 
importance for many applications. In order to sort out the key factors determining accuracy, we compared the predictions of 60 density functional approximations of 10 different types [local spin density approximation, generalized gradient approximation (GGA), nonseparable gradient approximation (NGA), global-hybrid GGA, range-separated hybrid GGA, range-separated hybrid GGA plus molecular mechanics, meta-GGA, meta-NGA, global hybrid meta-GGA, and rangeseparated hybrid meta-GGA] for their ability to represent the spin-flip transitions of all $4 \mathrm{~d}$ transition metal atoms of groups 3-10 (Y through $\mathrm{Pd}$ ) and their singly positive cations. We considered all 16 excitation energies connecting the ground states (of the neutral atoms and the cations) to their first excited states of different multiplicities, and we also consider all eight ionization potentials. We also tested the Hartree-Fock method. All density functional and Hartree-Fock calculations are converged to a stable solution, in which the spatial symmetry is allowed to be completely broken to achieve the lowest possible energy solution. By analyzing the fractional subshell occupancies and spin contaminations, we were able to sort out the effects of s orbital vs. d orbital bias and high-spin vs. low-spin bias. A reliable functional should have little or no bias of either type rather than succeeding for a limited subset of cases by cancellation of errors. We found that the widely used correlations of spin splittings to percentage of Hartree- Fock exchange are not borne out by the data, and the correlation functionals also play a significant role. We eventually concluded that SOGGA11$\mathrm{X}, \mathrm{B} 1 \mathrm{LYP}, \mathrm{B} 3 \mathrm{~V} 5 \mathrm{LYP}$, and MPW3LYP are the most consistently reliable functionals for balanced treatments of $4 \mathrm{~d}$ transition metal atoms and their cations.

A notorious failing of approximate exchange-correlation functionals when applied to problems involving catalysis has been the inability of most local functionals to predict the correct adsorption site for $\mathrm{CO}$ on metal surfaces or to simultaneously predict accurate surface formation energies and adsorption energies for transition metals. By adding the kinetic energy density $\mathrm{T}$ to the density functional, the revTPSS density functional was shown recently to achieve a balanced description of surface energies and adsorption energies. We showed that the older M06-L density functional, also containing T, provides improved surface formation energies and $\mathrm{CO}$ adsorption energies over revTPSS for five transition metals and correctly predicts the on-top/hollow site adsorption preferences for four of the five metals, which was not achieved by most other local functionals. Because M06-L was entirely designed on the basis of atomic and molecular energies, its very good performance is a confirmation of the reasonableness of its functional form. Two GGA functionals with an expansion in the reduced gradient that is correct through second order, namely, SOGGA and SOGGA11, were also tested and found to produce the best surface formation energies of all tested GGA functionals, although they significantly overestimate the adsorption energies.

We presented two new exchange-correlation functionals for hybrid Kohn-Sham electronic structure calculations based on the nonseparable functional form 
introduced recently in the N12 and MN12-L functionals but now with the addition of screened Hartree-Fock exchange. The first functional depends on the density and the density gradient and is called N12-SX; the second functional depends on the density, the density gradient, and the kinetic energy density and is called MN12-SX. Both new functionals include a portion of the Hartree-Fock exchange at short-range, but Hartree-Fock exchange is screened at long range. The accuracies of the two new functionals are compared to those of the recent N12 and MN12-L local functionals to show the effect of adding screened exchange, are compared to the previously best available screened exchange functional, HSE06, and are compared to the best available global-hybrid generalized gradient approximation (GGA) and to a high-performance long-range-corrected meta-GGA.

The new density functionals M11-L and M11 and the SMD implicit solvation model were used to predict reduction potential-pH equilibrium diagrams (Pourbaix diagrams) for two ruthenium-based water-oxidation catalysts and their derivatives in aqueous solution. The observed improved accuracy for transitionmetal complexes opens new opportunities for the use of theory in the understanding and design of catalysts containing transition metals.

We reported a test of 30 density functionals, including several recent ones, for their predictions of 69 singlet-to-singlet excitation energies of 11 molecules. The reference values are experimental results collected by Caricato et al. for 30 valence excitations and 39 Rydberg excitations. All calculations employ timedependent density functional theory in the adiabatic, linear-response approximation. As far as reasonable, all of the assignments are performed by essentially the same protocol as used by Caricato et al. , and this allows us to merge our mean unsigned errors (MUEs) with the ones they calculated for both density functional and wave function methods. We found 21 of the 30 density functionals calculated here have smaller MUEs for the 30 valence states than what they obtained $(0.47 \mathrm{eV})$ for the state-of-the-art EOM-CCSD wave function. In contrast, for all of density functionals the MUE for 39 Rydberg states is larger than that $(0.11 \mathrm{eV})$ of EOM-CCSD. Merging the 30 density functionals calculated here with the 26 calculated by Caricato et al. makes a set of 56 density functionals. Averaging the unsigned errors over both the valence excitations and the Rydberg excitations, none of the 56 density functionals shows a lower mean unsigned error than that $(0.27 \mathrm{eV})$ of EOM-CCSD. Nevertheless, two functionals are successful in having an overall mean unsigned error of $0.30 \mathrm{eV}$, and another nine are moderately successful in having overall mean unsigned errors in the range $0.32-0.36 \mathrm{eV}$. Successful or moderately successful density functionals include seven hybrid density functionals with $41 \%$ to $54 \%$ Hartree-Fock exchange, and four range-separated hybrid density functionals in which the percentage of Hartree-Fock exchange increases from $0 \%$ to $19 \%$ at small interelectronic separation to $65 \%-100 \%$ at long range.

Using quantum chemical approximations to understand and predict complex transition metal chemistry, such as catalytic processes and materials properties, 
is an important activity in modern computational chemistry. High-level theory can sometimes provide high-precision benchmarks for systems containing transition metals, and these benchmarks can be used to understand the reliability of less expensive quantum chemical approximations that are applicable to complex systems. We studied the ionization potential energy of $\mathrm{Fe}$ and $\mathrm{FeC}$ and the bond dissociation energies of $\mathrm{FeC}$ and $\mathrm{FeC}^{+}$by 15 density functional approximations: M05, M06, M06-L, w B97, w B97X, w B97X-D, T -HCTHhyb, BLYP, B3LYP, M08HX, M08-SO, SOGGA11, SOGGA11-X, M11, and M11-L. All of the functionals predict the correct spin state as the ground state of neutral iron atom, but five of them predict the wrong spin state for $\mathrm{Fe}^{+}$. In the final analysis, four functionals, namely M11-L, T-HCTHhyb, SOGGA11, and M06-L, have small mean unsigned errors when averaged over two bond dissociation energies and two ionization potentials. In fact, the results showed that M11-L gives the smallest averaged mean unsigned error, i.e., M11-L is the most reliable density functional for these iron carbide systems among those studied.

Kohn-Sham density functional theory is in principle an exact formulation of quantum mechanical electronic structure theory, but in practice we have to rely on approximate exchange-correlation (xc) functionals. The objective of our work has been to design an xc functional with broad accuracy across as wide an expanse of chemistry and physics as possible, leading - as a long-range goalto a functional with good accuracy for all problems, i.e. a universal functional. To guide our path towards that goal and to measure our progress, we have developed-building on earlier work of our group - a set of databases of reference data for a variety of energetic and structural properties in chemistry and physics. These databases include energies of molecular processes, such as atomization, complexation, proton addition and ionization; they also include molecular geometries and solid-state lattice constants, chemical reaction barrier heights, and cohesive energies and band gaps of solids. We gathered many of these databases into four comprehensive databases, two with 384 energetic data for chemistry and solid-state physics and another two with 68 structural data for chemistry and solid-state physics, and we test two wave function methods and 77 density functionals ( 12 Minnesota meta functionals and 65 others) in a consistent way across this same broad set of data. We especially highlighted the Minnesota density functionals, but the results have broader implications in that one may see the successes and failures of many kinds of density functionals when they are all applied to the same data. Therefore, the results provide a status report on the quest for a universal functional.

We presented a new approach to calculating potential energy surfaces for photochemical reactions by combining self-consistent-field calculations for singlereference ground and excited states with symmetry-corrected spin-flip Tamm-Dancoff approximation calculations for multireference electronic states. The method is illustrated by an application with the M05-2X exchange-correlation functional to cis-trans isomerization of the penta-2,4-dieniminium cation, which is a model (with three conjugated double bonds) of the protonated Schiff base of 
retinal. We found good agreement with multireference configuration interactionplus-quadruples (MRCISD+Q) wave function calculations along three key paths in the strong-interaction region of the ground and first excited singlet states.

Endohedral fullerenes have great potential for a variety of technological applications. We considered $\mathrm{B} @ \mathrm{C}_{60}$ and showed that the amount of charge transfer from the semimetal boron atom to the cage is a strong function of the radial distance of the atom from the center of the fullerene, and it is controlled by multistate conical intersections whose associated ridge of avoided crossings has the topology of a Euclidean sphere. The potential energy surfaces of $\mathrm{B} @ \mathrm{C}_{60}$ are characterized by two kinds of local minima: those with a boron atom located in the geometric center of the fullerene, and those with a boron atom bound to the fullerene inner wall. At the lowest-energy minimum, at the center, the boron atom is neutral, whereas the transition to the wall is accompanied by an electron transfer from boron to the fullerene cage. The two kinds of minima are separated by a ridge of avoided crossings that forms a surface with a nearly spherical shape. The properties of such systems may be altered by controlling the populations of the two kinds of minima, for example, by application of an external field. Such switchable atom-cage charge transfer may find applications in novel molecular devices.

The 3d-series transition metals (also called the fourth-period transition metals), Sc to $\mathrm{Zn}$, are very important in industry and biology, but they provide unique challenges to computing the electronic structure of their compounds. In order to successfully describe the compounds by theory, one must be able to describe their components, in particular the constituent atoms and cations. In order to understand the ingredients required for successful computations with density functional theory, it is useful to examine the performance of various exchangecorrelation functionals; we did this for $4 \mathrm{~s}^{N} 3 \mathrm{~d}^{N^{\prime}}$ transition-metal atoms and their cations. We analyzed the results using three ways to compute the energy of the open-shell states: the direct variational method, the weighted-averaged broken symmetry (WABS) method, and a new broken-symmetry method called the reinterpreted broken symmetry (RBS) method. We found the RBS method to be comparable in accuracy with the WABS method. By examining the overall accuracy in treating 18 multiplicity-changing excitations and 10 ionization potentials with the RBS method, 10 functionals were found to have a meanunsigned error of $<5 \mathrm{kcal} / \mathrm{mol}$, with $\omega B 97 X \mathrm{D}$ topping the list. For local density functionals, which are more practical for extended systems, the M06-L functional is the most accurate. And by combining the results with our previous studies of $p$ block and 4d-series elements as well as databases for alkyl bond dissociation, main-group atomization energies, and $\pi-\pi$ noncovalent interactions, we found five functionals, namely, PW6B95, MPW1B95, M08-SO, SOGGA11-X, and MPWB1K, to be highly recommended. We also studied the performance of PW86 and C09 exchange functionals, which have drawn wide interest in recent studies due to their claimed ability to reproduce Hartree- Fock exchange at long distance. By combining them with four correlation functionals, we found the 
performance of the resulting functionals disappointing both for $3 \mathrm{~d}$ transition-metal chemistry and in broader tests, and thus we do not recommend PW86 and C09 as components of generalized gradient approximations for general application.

When the spins of molecular orbitals are allowed to be aligned with different directions in space rather than being aligned collinearly, the resulting noncollinear spin orbitals add extra flexibility to variational optimization of the orbitals, and solutions obtained with collinear spin orbitals may be unstable with respect to becoming noncollinear in the expanded variational space. We explored whether and in what way the molecular orbitals of the Kohn- Sham density functional theory become noncollinear when fully optimized for multireference molecules, transition states, and reaction paths. (I note that a noncollinear determinant has intermediate flexibility between a collinear determinant and a linear combination of many collinear determinants with completely independent coefficients. However, the Kohn- Sham method is defined to involve the variational optimization of a single determinant, and a noncollinear determinant represents the limit of complete optimization in the Kohn-Sham scheme.) We compared the results obtained with the noncollinear Kohn- Sham (NKS) scheme to those obtained with the widely used unrestricted Kohn- Sham (UKS) scheme for two types of multi-reference systems. For the dissociation of the $\mathrm{MnO}$ and $\mathrm{NiO}$ transition metal oxides, we found UKS fails to dissociate to the ground states of neutral atoms, while NKS dissociates to the correct limit and predicts potential energy curves that vary smoothly at intermediate bond lengths. This is due to the instability of UKS solutions at large bond distances. For barrier heights of $\mathrm{O} 3, \mathrm{BeH} 2$, and $\mathrm{H} 4$, NKS is shown to stabilize the multi-reference transition states by expanding the variational space. Although the errors vary because they are closely coupled with the capability of the employed exchange-correlation functionals in treating the multiconfigurational states, these findings demonstrated that results with collinear spin orbitals should be further scrutinized, and the future development of exchangecorrelation functionals for multi-reference systems should incorporate the flexibilities of NKS.

It is important to test methods for simulating water, but small water clusters for which benchmarks are available are not very representative of the bulk. We presented benchmark calculations, in particular $\operatorname{CCSD}(T)$ calculations at the complete basis set limit, for water 26-mers drawn from Monte Carlo simulations of bulk water. These clusters are large enough that each water molecule participates in 2.5 hydrogen bonds on average. The electrostatically embedded three-body approximation with $\operatorname{CCSD}(\mathrm{T})$ embedded dimers and trimers reproduces the relative binding energies of eight clusters with a mean unsigned error (MUE, kcal per mole of water molecules) of only 0.009 and $0.015 \mathrm{kcal}$ for relative and absolute binding energies, respectively. Using only embedded dimers (electrostatically embedded pairwise approximation) raises these MUEs to 0.038 and $0.070 \mathrm{kcal}$, and computing the energies with the M11 exchangecorrelation functional, which is very economical, yields errors of only 0.029 and 


\section{$0.042 \mathrm{kcal}$.}

In order to understand what governs the accuracy of approximate exchangecorrelation functionals for intrinsically multiconfigurational systems containing metal atoms, the properties of the ground electronic state of $\mathrm{CaO}$ werestudied in detail. We first applied the T1, TAE(T), B1, and M diagnostics to $\mathrm{CaO}$ and confirmed that $\mathrm{CaO}$ is an intrinsically multiconfigurational system. Then, we compared the bond dissociation energies (BDEs) of $\mathrm{CaO}$ as calculated by 49 exchange- correlation functionals, three exchange-only functionals, and the HF method. To analyze the error in the BDEs for the various functionals, we decomposed each calculated BDE into four components, in particular the ionization potential, the electron affinity, the atomic excitation energy of the metal cation to prepare the valence state, and the interaction energy between prepared states. We found that the dominant error occurs in the calculated atomic excitation energy of the cation. Third, we compared dipole moments of $\mathrm{CaO}$ as calculated by the 53 methods, and we analyzed the dipole moments in terms of partial atomic charges to understand the contribution of ionic bonding and how it is affected by errors in the calculated ionization potential of the metal atom. We then analyzed the dipole moment in terms of the charge distribution among orbitals, and we found that the orbital charge distribution does not correlate well with the difference between the calculated ionization potential and electron affinity. Fourth, we examined the potential curves and internuclear distance dependence of the orbital energies of the lowest-energy $\mathrm{CaO}$ singlet and triplet states to analyze the near-degeneracy aspect of the correlation energy. The most important conclusion is that the error tends to be dominated by the error in the relative energies of $\mathrm{s}$ and $\mathrm{d}$ orbitals in $\mathrm{Ca}+$, and the most popular density functionals predict this excitation energy poorly. Thus, even if they were to predict the BDE reasonably well, it would be due to cancellation of errors. The effect of the cation excitation energy can be understood in terms of an orbital picture, as follows. For most functionals the predicted cation excitation energy is too small, so it is too easy to delocalize charge from the oxygen $2 p$ orbital to the $\mathrm{Ca}+\mathrm{d}$ orbital; this overestimates the covalency and explains why most functionals overestimate the bond energy.

Vanadium dimer is a notoriously difficult case for Kohn- Sham (KS) density functional theory with currently available approximations to the exchangecorrelation (xc) functionals, and many approximate xc functionals yield an exceedingly large error in the calculated bond energy. In this paper, we first test the bond energies estimated by $43 \mathrm{xc}$ functionals and the Hartree- Fock (HF) method. The results further confirmed the large errors and showed that, with the experimental bond energy being $64.2 \mathrm{kcal} / \mathrm{mol}$, the $\mathrm{KS}$ calculations give predictions all over the map with errors ranging from -61.5 to $+60.5 \mathrm{kcal} / \mathrm{mol}$, and the HF method performs much worse with an error of $-124.4 \mathrm{kcal} / \mathrm{mol}$ ! The reason for these very large errors was examined by analyzing the atomic and molecular orbital energies calculated by various xc functionals. The results showed that the errors in estimates of the bond energy of vanadium dimer can 
primarily be related to the calculated energy gap between the $4 s$ and $3 d_{z^{2}}$ atomic orbitals of the vanadium atom and especially to the $3 d_{z^{2}}$ orbital energy. This interesting relation between the errors in the calculated bond energy and the magnitudes of the single-particle orbital energies provides a constructive alternative to the common but more sterile explanation that it is the static correlation energy due to multicenter left-right correlation that makes the vanadium dimer and many other transition metal compounds so difficult for Kohn- Sham calculations. One of the most important factors in determining the critical atomic orbital energy is the amount of nonlocal HF exchange that is included in the $x c$ functional, but it is still difficult to explain why different local functionals (functionals with no HF exchange) yield quite different results. We concluded that improving calculations of orbital energies of atoms may provide a route to improving the accuracy of theoretical predictions of molecular bond energies for systems containing metal atoms.

We studied the $\mathrm{Co}_{4} \mathrm{O}_{4}$ subnanocluster and its $\mathrm{MeCN}$-coated species using density functional theory, and we found that the $\mathrm{Co}_{4} \mathrm{O}_{4}$ core presents distinctive structures in bare and ligand-coated species. We proposed a possible ligandmediated ring $\rightarrow$ cube transformation mechanism during the ligand-coating process of the $\mathrm{Co}_{4} \mathrm{O}_{4}$ core due to the stronger binding energies of the $\mathrm{MeCN}$ ligands to the 3D distorted cube structure than to the 2D ring and ladder structures; theory indicates that three ligands are sufficient to stabilize the cube structure. Both ring and cube structures are ferromagnetic. Our finding is potentially useful for understanding the catalysis mechanism of $\mathrm{Co}_{4} \mathrm{O}_{4}$ species, which have important applications in solar energy conversion and water splitting; these catalysis reactions usually involve frequent addition and subtraction of various ligands and thus possibly involve core rearrangement processes similar to our findings.

We presented a new theoretical framework, called Multiconfiguration PairDensity Functional Theory (MCPDFT), which combines multiconfigurational wave functions with a generalization of density functional theory (DFT). A multiconfigurational self-consistent-field (MCSCF) wave function with correct spin and space symmetry was used to compute the total electronic density, its gradient, the on-top pair density, and the kinetic and Coulomb contributions to the total electronic energy. We then used a functional of the total density, its gradient, and the on-top pair density to calculate the remaining part of the energy, which we call the on-top density-functional energy in contrast to the exchange-correlation energy of Kohn-Sham DFT. Because the on-top pair density is an element of the two-particle density matrix, this goes beyond the Hohenberg-Kohn theorem that refers only to the one-particle density. To illustrate the theory, we obtained first approximations to the required new type of density functionals by translating conventional density functionals of the spin densities using a simple prescription, and we performed post-SCF density functional calculations using the total density, density gradient, and on-top pair density from the MCSCF calculations. Double counting of dynamic correlation or 
exchange does not occur because the MCSCF energy is not used. The theory is illustrated by applications to the bond energies and potential energy curves of $\mathrm{H}_{2}$, $\mathrm{N}_{2}, \mathrm{~F}_{2}, \mathrm{CaO}, \mathrm{Cr}_{2}$, and $\mathrm{NiCl}$ and the electronic excitation energies of $\mathrm{Be}, \mathrm{C}, \mathrm{N}, \mathrm{N}^{+}$, $\mathrm{O}, \mathrm{O}^{+}, \mathrm{Sc}^{+}, \mathrm{Mn}, \mathrm{Co}, \mathrm{Mo}, \mathrm{Ru}, \mathrm{N}_{2}, \mathrm{HCHO}, \mathrm{C}_{4} \mathrm{H}_{6}, \mathrm{C}_{-} \mathrm{C}_{5} \mathrm{H}_{6}$, and pyrazine. The method presented has a computational cost and scaling similar to MCSCF, but a quantitative accuracy, even with the present first approximations to the new types of density functionals, that is comparable to much more expensive multireference perturbation theory methods.

Systematic evaluation of the accuracy of exchange-correlation functionals is essential to guide scientists in their choice of an optimal method for a given problem when using density functional theory. We determined the accuracy of one Generalized Gradient Approximation (GGA) functional, three meta-GGA functionals, one Nonseparable Gradient Approximation (NGA) functional, one meta-NGA, and three hybrid GGA functionals was evaluated for calculations of the closest interatomic distances, cohesive energies, and bulk moduli of all 3d, $4 \mathrm{~d}$, and $5 \mathrm{~d}$ bulk transition metals that have face centered cubic (fcc), hexagonal closed packed (hcp), or body centered cubic (bcc) structures (a total of 27 cases). Our results show that including the extra elements of kinetic energy density and Hartree- Fock exchange energy density into gradient approximation density functionals does not usually improve them. Nevertheless, the accuracies of the Tao-Perdew-Staroverov-Scuseria (TPSS) and M06-L meta-GGAs and the MN12-L meta- NGA approach the accuracy of the Perdew-Burke-Ernzerhof (PBE) GGA, so usage of these functionals may be advisable for systems containing both solid-state transition metals and molecular species. The N12 NGA functional is also shown to be almost as accurate as PBE for bulk transition metals, and thus it could be a good choice for studies of catalysis given its proven good performance for molecular species. 